\title{
Effect of antibiotic treatment on Oxalobacter formigenes colonization of the gut microbiome and urinary oxalate excretion
}

\section{Lama Nazzal}

New York University Langone Medical Center

Fritz Francois

New York University Langone Medical Center

Nora Henderson

New York University Langone Medical Center

Menghan Liu

New York University Langone Medical Center

\section{Huilin Li}

New York University Langone Medical Center

\section{Hyunwook Koh}

The State University of New York, Korea, Incheon, 21985, South Korea

Chan Wang

New York University Langone Medical Center

\section{Zhan Gao}

Rutgers, The State University of New Jersey

\section{Guillermo Perez}

New York University Langone Medical Center

John Asplin

litholink, corp.

David Goldfarb

New York University Langone Medical Center

Martin Blaser ( $\sim$ Martin.Blaser@cabm.rutgers.edu )

Rutgers, The State University of New Jersey

\section{Research Article}

Keywords: 0 . formigenes colonization, urine electrolytes

Posted Date: January 4th, 2021

DOl: https://doi.org/10.21203/rs.3.rs-131217/v1 
License: (c) (i) This work is licensed under a Creative Commons Attribution 4.0 International License. Read Full License

Version of Record: A version of this preprint was published at Scientific Reports on August 12th, 2021. See the published version at https://doi.org/10.1038/s41598-021-95992-7. 


\section{Abstract}

Background: The incidence of kidney stones is increasing in the US population. Oxalate, a major factor for stone formation, is degraded by gut bacteria reducing its intestinal absorption. Intestinal $O$. formigenes colonization has been associated with a lower risk for recurrent kidney stones in humans. In the current study, we used a clinical trial of the eradication of Helicobacter pylori to assess the effects of an antibiotic course on $\boldsymbol{O}$. formigenes colonization, urine electrolytes, and the composition of the intestinal microbiome.

Methods: Of 69 healthy adult subjects recruited, 19 received antibiotics for $H$. pylori eradication, while 46 were followed as controls. Serial fecal samples were examined for 0 . formigenes presence and microbiota characteristics. Urine, collected serially fasting and following a standard meal, was tested for oxalate and electrolyte concentrations.

Results: $O$. formigenes prevalence was $50 \%$. Colonization was significantly and persistently suppressed in antibiotic-exposed subjects but remained stable in controls. Urinary $\mathrm{pH}$ increased after antibiotics, but urinary oxalate did not differ between the control and treatment groups. The 0 . formigenes-positive samples had higher alpha-diversity and significantly differed in Beta-diversity from the

O. formigenes-negative samples. Specific taxa varied in abundance in relation to urinary oxalate levels.

Conclusions: These studies identified significant antibiotic effects on $O$. formigenes colonization and urinary electrolytes and showed that overall microbiome structure differed in subjects according to 0 . formigenes presence. Identifying a consortium of bacterial taxa associated with urinary oxalate may provide clues for the primary prevention of kidney stones in healthy adults.

\section{Introduction}

Nephrolithiasis (kidney stones) affect up to 9 percent of the US population[1], affecting both males and females, with incidence increasing in children and adults[2,3]. Most stones are composed of calcium oxalate $(\mathrm{CaOx})[4,5]$. Oxalate is absorbed in the gut from the diet and also produced endogenously as an end-product of amino acid metabolism[6, 7]. Human intestinal bacteria that degrade oxalate and contribute to its metabolism are considered the oxalobiome[8]. Increasing evidence suggests gut microbiota roles in nephrolithiasis pathogenesis [9-11].

Prior antibiotic use has been associated with kidney stone development months or even years later[12, 13]. Although multiple bacterial taxa are able to degrade oxalate in the gut $[8,14]$, Oxalobacter formigenes is the only commensal known to use oxalate as its sole energy and carbon source and may be the only specialist oxalate degrader in humans[15]. In rodent models, colonic colonization with 0 . formigenes significantly reduced urinary oxalate excretion[16-18]. Intestinal $O$. formigenes colonization has been associated with lower risk for recurrent kidney stones in humans[19]. 
Epidemiologic studies provide evidence that $O$. formigenes prevalence in developed countries such as the USA is lower than in developing countries $[20,21]$. $O$. formigenes is susceptible to commonly used antibiotics [22]; an antibiotic course for $H$. pylori eradication led to loss of colonization for at least six months post-treatment [23], but post-treatment examination of urinary analytes and microbiome were not done.

In the current study, we used a study (ESSAY) of the eradication of Helicobacter pylori to assess the effect of an antibiotic course on $O$. formigenes colonization and on urine electrolytes, including oxalate excretion. We also asked whether microbiome structure and composition differed over time with respect to both 0 . formigenes status and antibiotic exposure.

\section{Results}

\section{Recruitment}

Of 69 eligible participants who returned for the baseline visit, 23 (33\%) tested positive for $H$. pylori while 46 tested negative; 65 of the 69 participants also provided fecal samples at the baseline visit. H. pyloripositive participants $(n=19)$ who consented to the antibiotic treatment received antibiotics based on the standard-of-care. Of those, 17 completed the final study visit at 6 months. Four subjects who underwent a baseline assessment and tested positive for H.pylori withdrew from the study before receiving any antibiotics (Fig. 1).

\section{O. formigenes colonization status and stability over time.}

Based on the $O$. formigenes positivity score, the prevalence of 0 . formigenes was $50 \%$ in the 64 participants at baseline (Fig. 1,2), similar to prior findings in the USA [19, 21, 26, 29]. The 0 . formigenespositive and negative participants were similar in distributions of age, sex, ethnicity, BMI, mode of birthdelivery, $H$. pylori status, and place of birth (Table S2). We evaluated $O$. formigenes colonization status stability over time in both the antibiotic-treated participants and controls. Among the controls, of the 210 . formigenes-positive subjects at baseline assessed at 6 weeks, 19 (90\%) remained positive, vs. 0/20 formigenes-negative participants at baseline becoming positive $(p<0.001)$. In contrast, in the antibiotictreated group, of the 8 initially 0 . formigenes-positive participants, only $2(25 \%)$ remained positive at 6 weeks vs. $19 / 20$ (95\%) who were untreated, and only three (37.5\%) at 24 weeks vs. $20 / 24(83.3 \%)$ in the untreated controls. All 11 of the baseline $O$. formigenes-negative group remained negative (Fig. 2A, B). Thus, treatment to eradicate $H$. pylori had a strong effect, largely eliminating $O$. formigenes, confirming prior studies [23].

0 . formigenes detection by high throughput 16S rRNA sequencing. Our prior studies showed that $O$. formigenes abundance can vary in carriers over a range $>3-\log _{10}$, with some individuals having minimally detectable numbers $[26,27]$, which intrinsically limits interpreting positivity. 
Using 16S rRNA sequencing, we detected 0 . formigenes in 10 (22\%) of the 45 controls at baseline, vs. $24(53 \%)$ using the above 0 . formigenes scoring system. At follow-up, we detected 0 . formigenes in six other controls by $16 \mathrm{~S}$ analysis, but positivity fluctuated for individual subjects (Fig. 3). However, across all the control subjects, the mean \% relative abundance was stable over time $(0.06 \pm 0.0$ at baseline and $0.03 \pm 0.04$ at week 24). At baseline in the antibiotic-treated group, 0 . formigenes was detected in 4 (21.1\%) of 19 participants; after antibiotic treatment, 0 . formigenes was significantly and persistently suppressed in all four.

Urinary Parameters according to antibiotic treatment and O. formigenes status.

The urinary oxalate to creatinine ratio $(\mathrm{UOx} / \mathrm{Cr} \mathrm{mg} / \mathrm{g})$ before and after the standard meal was measured at baseline and follow-up visits in the antibiotic-treated participants and untreated controls. In both the controls and treatment group, pre- and post-meal Uox/Cr did not change over time ( $p>0.1)$ (Suppl Fig. 2). Urine $\mathrm{pH}$ levels were unchanged in the untreated controls, as expected, but increased in the treated patients in both the pre- and post-meal comparisons ( $p=0.04$ and 0.06 , respectively). Urine ammonia/Cr differed in their direction of change in controls and treated subjects in pre-meal samples but after the meal increased in both control and antibiotic-treated participants. Urine citrate/Cr was stable over time in pre-meal samples from controls and in post-meal samples from both controls and treated subjects but significantly increased in the treatment group. The ratio to creatinine of urinary sodium, phosphorus, calcium, and urea nitrogen increased pre-meal in both the control and antibiotic-treated subjects, but were unchanged in post-meal comparisons over time (Table S3). These results indicate the effects of antibiotic exposures on urinary electrolyte excretion, pertaining particularly to acid-base homeostasis.

\section{Microbial Community structure}

Alpha-diversity is used to measure the bacterial diversity within a sample, whereas Beta-diversity provides a measure of the dissimilarity between samples. First, we asked whether the microbiome differed in the study subjects according to $O$. formigenes status. Fecal samples from the $O$. formigenes-positive subjects had higher a-diversity $(\mathrm{p}<0.01)$ than those from 0 . formigenes-negative subjects (Fig. 3A). As expected, in the untreated controls, a-diversity remained stable, but after antibiotic treatment, decreased at 6 weeks and later returned to baseline (Fig. 4A). To assess the communities for $\beta$-diversity, we examined UniFrac distances in 173 samples from 64 participants according to 0 . formigenes baseline-status; $\beta$-diversity significantly differed in the samples based on 0 . formigenes status (Fig. 3B). Similar to a-diversity, $\beta$ diversity was stable over time in untreated controls, but changed $(p=0.06)$ immediately following antibiotic exposure, then returning to baseline (Fig. 4B). These studies provide evidence that $O$. formigenes status correlates with and clinical antibiotic regimen affects overall microbiome community characteristics.

\section{Association between $\mathrm{UOX} / \mathrm{Cr}$ and specific bacterial taxa}

To assess whether any taxa other than 0 . formigenes were directly or inversely associated with $\mathrm{UOx} / \mathrm{Cr}$, we first analyzed the controls (41 participants with 108 measurements at four time points), using a centered log-ratio (CLR) transformation to standardize relative abundances of individual microbial taxa 
before fitting to a linear mixed model (Table 1). The analyses showed that relative abundance of the phylum Proteobacteria was directly associated with urinary oxalate levels. Genus Dysgonomonas and species $D$. gadei were inversely associated with post-meal urinary oxalate level, adjusted for age, gender, ethnicity, BMI, and calcium level. Based on our model, in the antibiotic-treated subjects (Table 2), the clr transformed relative abundance of genera Dysgonomonas, Epulopiscium, and Providencia and species Bacteroides plebeius were inversely associated with pre-meal urinary oxalate level. whereas genus Lachnobacterium and species Faecalitalea cylindroides were directly associated. Family Barnesiellaceae and species Streptococcus gordonii were inversely associated with post-meal urinary oxalate levels, whereas order Turicibacterales and family Turicibacteraceae were directly associated.

\section{Discussion}

In this study, we confirmed that antibiotic treatment has a strong effect on 0 . formigenes status $[23,30]$. Although most antibiotic-treated participants had durable suppression, lasting $>24$ weeks, 0 . formigenes persisted in some, suggesting either heterogeneity in antibiotic resistance of 0 . formigenes strains or inability of the active agent(s) to achieve sufficient colonic concentrations. Since $O$. formigenes colonization has been associated with lower oxaluria[31], our observation of treatment effects provides one explanation for why antibiotic treatments have been associated with a higher kidney stone incidence[12, 32].

This study highlights the difficulty in assessing patients' $O$. formigenes status due to the wide range in colonization density that may reflect both diet and competing organisms. This variation confirms our prior work [26, 27], which provided the rationale for using a formula based on multiple tests to detect colonization. This biological variation is likely also in part responsible for the continued uncertainty about whether $\boldsymbol{O}$. formigenes status is associated with nephrolithiasis or not. Our results may more accurately represent colonization of individual subjects than previously since prior studies used a single assessment types done cross-sectionally, which can underestimate colonization prevalence. Giving subjects an oxalate-rich diet that selects for 0 . formigenes growth may enhance detection in future cross-sectional analyses. Finding that $16 \mathrm{~S}$ high throughput sequencing has low sensitivity for $O$. formigenes detection, due to its often low abundance, may be useful in future studies where colonization needs to be confirmed using more sensitive methods.

The standard meal used in this study did not contain a substantial oxalate load because the original trial was not designed to measure our primary outcome. Therefore, urinary oxalate changes might not reach those observed with moderate-high oxalate diets that healthy individuals typically consume. Differences in $\mathrm{UOx} / \mathrm{Cr}$ (indicated by the standard deviations in Suppl Fig. 2) emphasize the need for larger trials with high oxalate, low calcium, and controlled diets. However, urinary $\mathrm{pH}$ increased after antibiotic treatment but not in controls, indicating possible effects beyond oxalate metabolism affecting acid-base homeostasis. The recent associations between increased stone risk and antibiotic courses did not include urinary chemistry data in one report[12], and in the second, the temporal relationship between antibiotic use and urine collection was not standardized [13]. Our findings provide potential mechanisms for the 
linkage between prior antibiotic use and nephrolithiasis risk. Higher urinary $\mathrm{pH}$ is associated with increased risk for calcium phosphate stones[33].

Our community structure findings indicate that $O$. formigenes presence is a marker for a richer microbiome (consistent with prior studies $[20,26]$ ) and its loss is a marker of important antibiotic-induced microbiome alterations. That no significant recolonization was detected by 6 months, while the overall diversity recovered, is consistent with persistent microbiome disturbance.

We identified a group of bacteria directly or inversely correlated with urinary oxalate level, consistent with the hypothesis that oxalate degradation in the gut is affected by the putative oxalobiome that facilitates oxalate degradation [8]. This study provides the first analysis of such associations in healthy subjects to potentially identify a microbiome associated with differential potential for stone formation. Since our findings indicate taxa with abundance associated with lower urinary oxalate levels, these could be protective against hyperoxaluria; species from genus Providencia are known to be oxalate-degraders[34]. Similarly, we identified bacteria associated with higher urinary oxalate levels whose presence could potentially increase kidney stone risk [9]. Proteobacteria were associated with higher urine oxalate in our study and were enriched in stone formers in two other studies $[9,11]$.

The lack of an association of $O$. formigenes colonization with lower urinary oxalate excretion could reflect its very low abundance in subjects without dietary oxalate enrichment and insensitive detection using 16S rRNA sequencing. Future studies with deeper coverage will facilitate identifying low-abundance bacterial taxa.

Our study was limited by the small sample size for the antibiotic-treated participants and lack of information about baseline diets. The standard meal did not contain oxalate, and the 3-hour post-meal urine collection did not permit study of less acute colonic bacterial actions on dietary components.

In conclusion, our study found substantial antibiotic effects on $\mathcal{O}$. formigenes colonization, its relationship with other taxa and overall microbiome community structure, and with several urinary parameters, and identified a consortium of bacterial taxa associated with urinary oxalate levels. In the future, understanding metabolic activities of the intestinal taxa may be useful for primary kidney stone prevention.

\section{Materials And Methods}

Recruitment and subject enrollment. Participants were recruited for the ESSAY study (Eradication Study in Stable Adults/Youths) evaluating the effect of the standard-of-care practice of $H$. pylori eradication on metabolic profile and anthropometric measures of healthy adults. Participants were identified from the Bellevue Hospital primary care clinic, and community. Healthy young adults who were 18-40 years old were screened by research coordinators for eligibility criteria and then signed an informed consent if meeting those criteria. In total, 139 participants were screened for participation. Of the 87 participants who met the eligibility requirements and provided informed consent, 69 completed the baseline visit while 
18 were lost to follow-up (Fig. 1). The clinical study was conducted between April 2012 and July 2016. We excluded participants with diabetes, hyper- or hypothyroidism, prior gastric or bariatric surgery, prior $H$. pylori treatment, steroid or other immunomodulatory drug use within 4 weeks of the first visit, and antibiotic use within the prior 6 months. Participants completed baseline questionnaires to provide their demographic information, medical history, and current medication use. The study was approved by the Institutional Review Board (IRB) at NYU Langone Health. All research was performed in accordance with the Declaration of Helsinki and our local IRB guidelines. Informed consent was obtained from all participants.

Determination of $\mathrm{H}$. pylori status at baseline and at follow-up and antibiotics regimen. Subjects underwent a non-radioactive ${ }^{13} \mathrm{C}$ Urea Breath Test (Meretek Diagnostics, New York NY) to determine H.pylori status. Subjects $(\mathrm{n}=23)$ who tested H. pylori-positive were offered treatment with a 14-day twicedaily regimen [amoxicillin $1000 \mathrm{mg}$, clarithromycin $500 \mathrm{mg}$, and proton pump inhibitor (PPl; omeprazole $20 \mathrm{mg}$, rabeprazole or esomeprazole $40 \mathrm{mg}$ )], per the then-current standard of care [24]. In total, 19 subjects received a course of antibiotics, including three who received a second antibiotic course because they failed eradication with the first course, and four other subjects withdrew from the study before receiving antibiotics (Fig. 1). The 46 subjects who were H.pylori negative at baseline did not receive antibiotics and were followed serially as controls.

Study time points and assessments. Subjects fasted overnight at home and then underwent basic assessment at the NYU Clinical and Translational Science Institute (CTSI) at Bellevue Hospital at the baseline and 6, 12, and 24-week timepoints. And height and weight obtained. Stool was collected at home the day prior to the assessments using a stool collection kit, brought to NYU, and stored immediately at $-80^{\circ} \mathrm{C}$. In total, we obtained fecal samples from 65 subjects at baseline (Fig. 1).

Test meal and urine collections. To assess the differences in urinary electrolytes before and after $H$. pylori eradication, we collected a fasting urine sample and then administered a standard 16-oz liquid meal totaling 700 calories ( 2 cans of Ensure Plus ${ }^{\circledR}$ ) (Table S1). Urine was collected for three hours after the meal. In total, we collected urine samples (pre and post) from 32 controls and 14 treated subjects, (Fig. 1).

DNA isolation and PCR. DNA was extracted from fecal samples and PCR was performed as described[21].

Quantitative PCR. qPCR was performed using the LightCycler 480 SYBR Green I Master Mix, primers targeting $O x c$, in the LightCycler 480 system (Roche, Pleasanton CA). The oligonucleotide primers were: forward, 5'-GTGTTGTCGGCATTCCTATC-3' and reverse 5'-GAAGCAGTTGGTGGTTGC-3'. Melting peak analysis was performed from $65^{\circ} \mathrm{C}$ to $95^{\circ} \mathrm{C}$ to confirm amplicon specificity. A positive result was defined by amplification greater than $1.0 \mathrm{E} 2$, with melting peak between $86-87^{\circ} \mathrm{C}$.

16S rRNA sequencing. For amplicon library generation, the V4 region of the $16 \mathrm{~S}$ rRNA gene was amplified with gene-specific primers, as described[21]. The 254 bp V4 region was sequenced using the Illumina 
MiSeq $2 \times 150$ bp platform at NYU Langone Health. Operational taxonomic units (OTUs) were picked using GreenGenes 13_8 for reference, using the DADA2 pipeline [25].

Sample diversity analyses. Intra-sample a-diversity was calculated using QIIME2, using phylogenetic diversity, observed OTU number, Chao1, and Shannon indices at rarefaction depths of 8,000 sequences/sample. Beta-diversity was assessed using the unweighted UniFrac distance metric [26].

Oxalobacter formigenes status determination. We tested each fecal sample for the presence of $O$. formigenes using PCR in duplicate, qPCR, and 16S rRNA sequencing. Based on the known several logfold biological variations in $O$. formigenes abundance in human fecal specimens[26, 27], given the ascertainment variability using different methods, we developed a positivity score based on the 42 control subjects who did not receive antibiotics and who were tested at baseline and at least once in follow-up. The maximum number of assessments for each test subject was 16 if the participant was tested at all 4 time points (baseline, weeks $6,12,24$ ) in all four assays; and a minimum of 8 if only tested at two time points. For the 42 control participants, the mean \pm SD number of determinations was $14.7 \pm$ 2.6. We computed the positivity score by dividing the number of positive assessments by the total number of assessments for that subject. Since the results were bimodal, as expected (Suppl. Figure 1) we assigned baseline 0 . formigenes status as negative with score $\leq 0.2$ and positive with score $\geq 0.4$. Only one subject had a score of 0.3 , which we considered indeterminate and we removed this participant from further analysis (Suppl. Figure 1).

Urine testing. Urine aliquots were mixed with either $\mathrm{HCl}$ (for oxalate measurements) or with thymol (for all other assessments) and then stored at $-80^{\circ} \mathrm{C}$. Pre-meal (fasting) and the 3-hour post-meal urinary samples were analyzed by Litholink Corporation (Chicago IL). In each urine sample, we measured calcium, chloride, creatinine, magnesium, sodium, potassium, phosphate, and ammonium concentrations by standard techniques by Beckman Synchron AU680 (Beckman Instruments, Brea CA), as described [28]; $\mathrm{pH}$ was measured by glass electrode. Oxalate was measured by enzyme assay using oxalate oxidase (Trinity Biotech, Bray, Ireland). Citrate was measured by enzyme assay using citrate lyase (Mannheim Bohringer, Mannheim, Germany). All urine parameters except $\mathrm{pH}$ were normalized by dividing by creatinine concentration to account for hydration status.

Longitudinal analysis on urine parameters. We compared urine parameters and their changing rates according to the subjects' baseline treatment status (control, treatment group) and $O$. formigenes colonization status during the period of $0-24$ weeks. A linear mixed model was fitted to data over that time interval in which the changing rates in urine parameters were compared. The covariates age, sex, and body mass index (BMI) were adjusted in the model.

Association between Uox/Cr and bacterial taxa. We used the linear mixed effect model to identify which microbial taxa were associated with pre/post-meal urinary oxalate level, adjusted for age, gender, ethnicity, BMI, and calcium level. We included data for the participants in (i) the control group, we analyzed a total of 108 pairs of microbial measurements and urinary oxalate level (pre-/post- dietary) at 
baseline, 6, 12 and 24 weeks; and (ii), in the antibiotic-treatment group, we analyzed a total of 51 pairs of microbial measurements and urinary oxalate level (pre-/post- dietary) at 6, 12, and 24 weeks. In the mixed effects model, intercept and slope of the linear time trend for each subject are regarded as random effects. ANOVA results indicate that no correlation exists between random intercept and slope. We used the pre- and post-meal urinary oxalate levels as a dependent variable, respectively, while the relative abundance of individual microbial taxa after centered log-ratio (CLR) transformation as the independent variable. To calculate adjusted $p$-values, the Benjamini-Hochberg procedure was applied for each taxonomic level. Unclassified microbial taxa were not included in the analyses.

\section{Declarations}

\section{Author contributions:}

LN wrote the main manuscript text and prepared the figures. FF, MJB, DSG, GPP, and LN designed the study. $\mathrm{LN}$ and $\mathrm{NH}$ performed the study. LN, ML, HL, HK, CW, and ZG performed the analysis. JRA tested the urine samples. All authors reviewed the manuscript.

\section{Acknowledgments.}

Supported in part by R01 DK090989, R01DK110014, the NYU CTSA grant UL1TR000038 from the National Center for the Advancement of Translational Science (NCATS), and U01 Al22245, all from the $\mathrm{NIH}$, the Oxalosis and Hyperoxaluria Foundation-American Society of Nephrology Career Development Award (to L.N.), the C\&D and Zlinkoff Funds, and the Foundation LeDucq Transatlantic Network. This study also was supported by Rare Kidney Stone Consortium grant U54KD083908, a part of the Rare Diseases Clinical Research Network, an initiative of the Office of Rare Diseases Research, the National Center for Advancing Translational Sciences (NCATS), and funded through a collaboration between the NCATS and NIDDK.

\section{References}

1. Scales, C.D., et al., Prevalence of kidney stones in the United States. Eur Urol, 2012. 62(1): p. 160-5.

2. Kittanamongkolchai, W., et al., The changing incidence and presentation of urinary stones over 3 decades. Mayo Clinic Proceedings, 2017. 93(3): p. 291-9.

3. Tasian, G.E., et al., Annual Incidence of Nephrolithiasis among Children and Adults in South Carolina from 1997 to 2012. Clin J Am Soc Nephrol, 2016. 11(3): p. 488-96.

4. Lieske, J.C., et al., Renal stone epidemiology in Rochester, Minnesota: an update. Kidney Int, 2006. 69(4): p. 760-4.

5. Lieske, J.C., et al., Stone composition as a function of age and sex. Clin J Am Soc Nephrol, 2014. 9(12): p. 2141-6. 
6. Holmes, R.P., H.O. Goodman, and D.G. Assimos, Dietary oxalate and its intestinal absorption. Scanning Microsc, 1995. 9(4): p. 1109-18; discussion 1118-20.

7. Holmes, R.P., Oxalate synthesis in humans: assumptions, problems, and unresolved issues. Mol.Urol., 2000. 4(4): p. 329-332.

8. Abratt, V.R. and S.J. Reid, Oxalate-degrading bacteria of the human gut as probiotics in the management of kidney stone disease. Adv Appl Microbiol, 2010. 72: p. 63-87.

9. Stern, J.M., et al., Evidence for a distinct gut microbiome in kidney stone formers compared to nonstone formers. Urolithiasis, 2016. 44(5): p. 399-407.

10. Ticinesi, A., et al., Understanding the gut-kidney axis in nephrolithiasis: an analysis of the gut microbiota composition and functionality of stone formers. Gut, 2018. 67(12): p. 2097-2106.

11. Suryavanshi, M.V., et al., Hyperoxaluria leads to dysbiosis and drives selective enrichment of oxalate metabolizing bacterial species in recurrent kidney stone endures. Sci Rep, 2016. 6: p. 34712.

12. Tasian, G.E., et al., Oral Antibiotic Exposure and Kidney Stone Disease. J Am Soc Nephrol, 2018.

13. Ferraro, P.M., et al., Antibiotic Use and Risk of Incident Kidney Stones in Female Nurses. Am J Kidney Dis, 2019.

14. Mogna, L., et al., Screening of different probiotic strains for their in vitro ability to metabolise oxalates: any prospective use in humans? J Clin Gastroenterol, 2014. 48 Suppl 1: p. S91-5.

15. Allison, M.J., et al., Oxalobacter formigenes gen. nov., sp. nov.: oxalate-degrading anaerobes that inhabit the gastrointestinal tract. Arch Microbiol, 1985. 141(1): p. 1-7.

16. Canales, B.K. and M. Hatch, Oxalobacter formigenes colonization normalizes oxalate excretion in a gastric bypass model of hyperoxaluria. Surg Obes Relat Dis, 2017. 13(7): p. 1152-1157.

17. Li, X., et al., Response of germ-free mice to colonization with 0 . formigenes and altered Schaedler flora. Appl Environ Microbiol, 2016. 82(23): p. 6952-60.

18. Hatch, M., et al., Oxalobacter sp. reduces urinary oxalate excretion by promoting enteric oxalate secretion. Kidney Int, 2006. 69(4): p. 691-8.

19. Kaufman, D.W., et al., Oxalobacter formigenes may reduce the risk of calcium oxalate kidney stones. J Am Soc Nephrol, 2008. 19(6): p. 1197-203.

20. Clemente, J.C., et al., The microbiome of uncontacted Amerindians. Sci Adv, 2015. 1(3): p. e1500183.

21. PeBenito, A., et al., Comparative prevalence of Oxalobacter formigenes in three human populations. Sci Rep, 2019. 9(1): p. 574.

22. Lange, J.N., et al., Sensitivity of human strains of Oxalobacter formigenes to commonly prescribed antibiotics. Urology, 2012. 79(6): p. 1286-9.

23. Kharlamb, V., et al., Oral antibiotic treatment of Helicobacter pylori leads to persistently reduced intestinal colonization rates with Oxalobacter formigenes. J Endourol, 2011. 25(11): p. 1781-5.

24. Chey, W.D., et al., ACG Clinical Guideline: Treatment of Helicobacter pylori Infection. Am J Gastroenterol, 2017. 112(2): p. 212-239. 
25. Bolyen, E., et al., Reproducible, interactive, scalable and extensible microbiome data science using QIIME 2. Nat Biotechnol, 2019. 37(8): p. 852-857.

26. Liu, M., et al., Oxalobacter formigenes-associated host features and microbial community structures examined using the American Gut Project. Microbiome, 2017. 5(1): p. 108-25.

27. Barnett, C., et al., The Presence of Oxalobacter formigenes in the Microbiome of Healthy Young Adults. J Urol, 2016. 195(2): p. 499-506.

28. Asplin, J., et al., Supersaturation and stone composition in a network of dispersed treatment sites. J Urol., 1998. 159(6): p. 1821-1825.

29. Kelly, J.P., et al., Factors related to colonization with Oxalobacter formigenes in U.S. adults. J Endourol, 2011. 25(4): p. 673-9.

30. Pebenito, A.M., et al., Development of a Humanized Murine Model for the Study of Oxalobacter formigenes Intestinal Colonization. J Infect Dis, 2019. 220(11): p. 1848-1858.

31. Siener, R., et al., The role of Oxalobacter formigenes colonization in calcium oxalate stone disease. Kidney Int, 2013. 83(6): p. 1144-9.

32. Nazzal, L. and M.J. Blaser, Does the Receipt of Antibiotics for Common Infectious Diseases Predispose to Kidney Stones? A Cautionary Note for All Health Care Practitioners. J Am Soc Nephrol, 2018. 29(6): p. 1590-1592.

33. Gault, M.H., et al., Comparison of patients with idiopathic calcium phosphate and calcium oxalate stones. Medicine (Baltimore), 1991. 70(6): p. 345-59.

34. Hokama, S., et al., Oxalate-degrading Providencia rettgeri isolated from human stools. Int J Urol, 2005. 12(6): p. 533-8.

\section{Tables}

Table 1. Microbial taxa associated with pre/post-dietary urinary oxalate levels in untreated controls*

\begin{tabular}{|c|c|c|c|c|c|c|c|c|}
\hline \multirow[t]{2}{*}{ Microbial Taxon } & \multicolumn{4}{|c|}{ Pre-meal urinary oxalate level } & \multicolumn{4}{|c|}{ Post-meal urinary oxalate level } \\
\hline & $\begin{array}{l}\text { Coef. } \\
\text { Est. }\end{array}$ & $\begin{array}{l}\text { Std. } \\
\text { Error }\end{array}$ & $\begin{array}{l}\text { Unadj. } \\
\text { p }\end{array}$ & $\begin{array}{l}\text { Adj. } \\
\text { p }\end{array}$ & $\begin{array}{l}\text { Coef. } \\
\text { Est. }\end{array}$ & $\begin{array}{l}\text { Std. } \\
\text { Error }\end{array}$ & $\begin{array}{l}\text { Unadj. } \\
\text { p }\end{array}$ & $\begin{array}{l}\text { Adj. } \\
\mathrm{p}\end{array}$ \\
\hline $\begin{array}{l}\text { Phylum } \\
\text { Proteobacteria }\end{array}$ & 0.02 & 0.04 & 0.01 & 0.05 & 0.01 & 0.01 & 0.54 & 0.94 \\
\hline $\begin{array}{l}\text { Genus } \\
\text { Dysgonomonas }\end{array}$ & -0.04 & 0.05 & 0.51 & 0.94 & -0.04 & 0.01 & $<0.01$ & $<0.01$ \\
\hline Dysgonomonas gadei & -0.02 & 0.05 & 0.67 & 0.96 & -0.05 & 0.01 & $<0.01$ & $<0.01$ \\
\hline
\end{tabular}

*The estimated regression coefficients, standard errors, and unadjusted/adjusted p-values for the effect of the relative abundance of individual microbial taxa with centered log-ratio transformation in fitted mixed effect models. Analysis adjusted for age, gender, ethnicity, BMI, and calcium level. 
Table 2. Microbial taxa associated with pre/post-dietary urinary oxalate level in antibiotic-treated subjects*

\begin{tabular}{|c|c|c|c|c|c|c|c|c|}
\hline \multirow[t]{2}{*}{ Microbial Taxon } & \multicolumn{4}{|c|}{ Pre-meal urinary oxalate level } & \multicolumn{4}{|c|}{ Post-meal urinary oxalate level } \\
\hline & $\begin{array}{l}\text { Coef. } \\
\text { Est. }\end{array}$ & $\begin{array}{l}\text { Std. } \\
\text { Error }\end{array}$ & $\begin{array}{l}\text { Unadj. } \\
\mathrm{p}\end{array}$ & Adj. p & $\begin{array}{l}\text { Coef. } \\
\text { Est. }\end{array}$ & $\begin{array}{l}\text { Std. } \\
\text { Error }\end{array}$ & $\begin{array}{l}\text { Unadj. } \\
\mathrm{p}\end{array}$ & $\begin{array}{l}\text { Adj. } \\
\text { p }\end{array}$ \\
\hline $\begin{array}{l}\text { Order } \\
\text { Turicibacterales }\end{array}$ & 0.05 & 0.02 & 0.05 & 0.31 & 0.02 & 0.01 & $<0.01$ & 0.01 \\
\hline $\begin{array}{l}\text { Family } \\
\text { Barnesiellaceae }\end{array}$ & -0.10 & 0.04 & 0.04 & 0.50 & -0.04 & 0.01 & $<0.01$ & 0.01 \\
\hline $\begin{array}{l}\text { Family } \\
\text { Turicibacteraceae }\end{array}$ & 0.04 & 0.02 & 0.08 & 0.60 & 0.02 & 0.01 & $<0.01$ & 0.01 \\
\hline $\begin{array}{l}\text { Genus } \\
\text { Dysgonomonas }\end{array}$ & -0.40 & 0.11 & $<0.01$ & 0.02 & -0.04 & 0.02 & 0.03 & 0.25 \\
\hline Genus Epulopiscium & -0.43 & 0.12 & $<0.01$ & 0.02 & -0.05 & 0.02 & 0.03 & 0.25 \\
\hline $\begin{array}{l}\text { Genus } \\
\text { Lachnobacterium }\end{array}$ & 0.08 & 0.01 & $<0.01$ & 0.02 & 0.01 & $<0.01$ & 0.03 & 0.25 \\
\hline Genus Providencia & -0.43 & 0.12 & $<0.01$ & 0.02 & -0.05 & 0.02 & 0.02 & 0.25 \\
\hline Bacteroides plebeius & -0.12 & 0.03 & $<0.01$ & 0.03 & -0.01 & $<0.01$ & 0.21 & 0.72 \\
\hline $\begin{array}{l}\text { Streptococcus } \\
\text { gordonii }\end{array}$ & -0.07 & 0.06 & 0.22 & 0.78 & -0.04 & 0.01 & $<0.01$ & 0.05 \\
\hline $\begin{array}{l}\text { Faecalitalea } \\
\text { cylindroides }\end{array}$ & 0.24 & 0.05 & $<0.01$ & $<0.01$ & 0.02 & 0.02 & 0.33 & 0.77 \\
\hline
\end{tabular}

\section{Figures}


Enrolled Subject Status

HP Positive (Treatment)

HP Negative (controls)

\section{Screened for Participation}

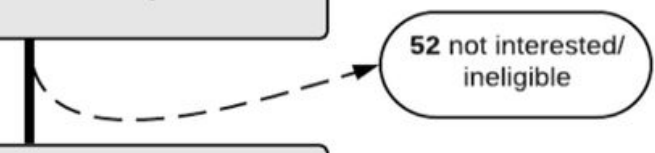

87 Completed HP Screening Visit

$24(28 \%)$ HP Positive

63 (72\%) HP Negative

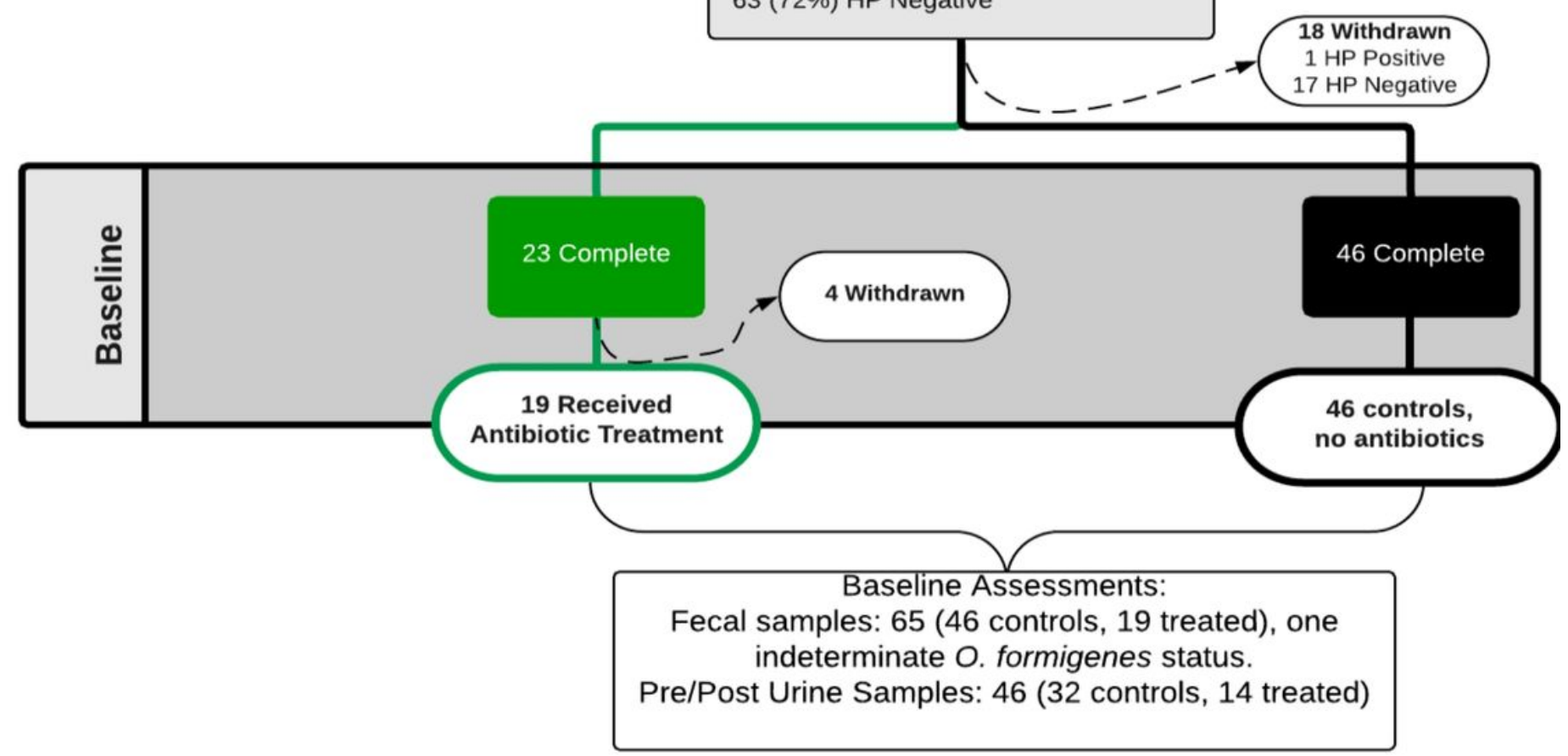

Figure 1

Study design and enrollment. Of 139 subjects screened for participation, 87 completed the screening, and 69 subjects underwent baseline assessment. 19 H.pylori-positive subjects were treated with antibiotics ,whereas 46 H.pylori-negative subjects served as controls. 
Fig. 2

\section{A: Control subjects}

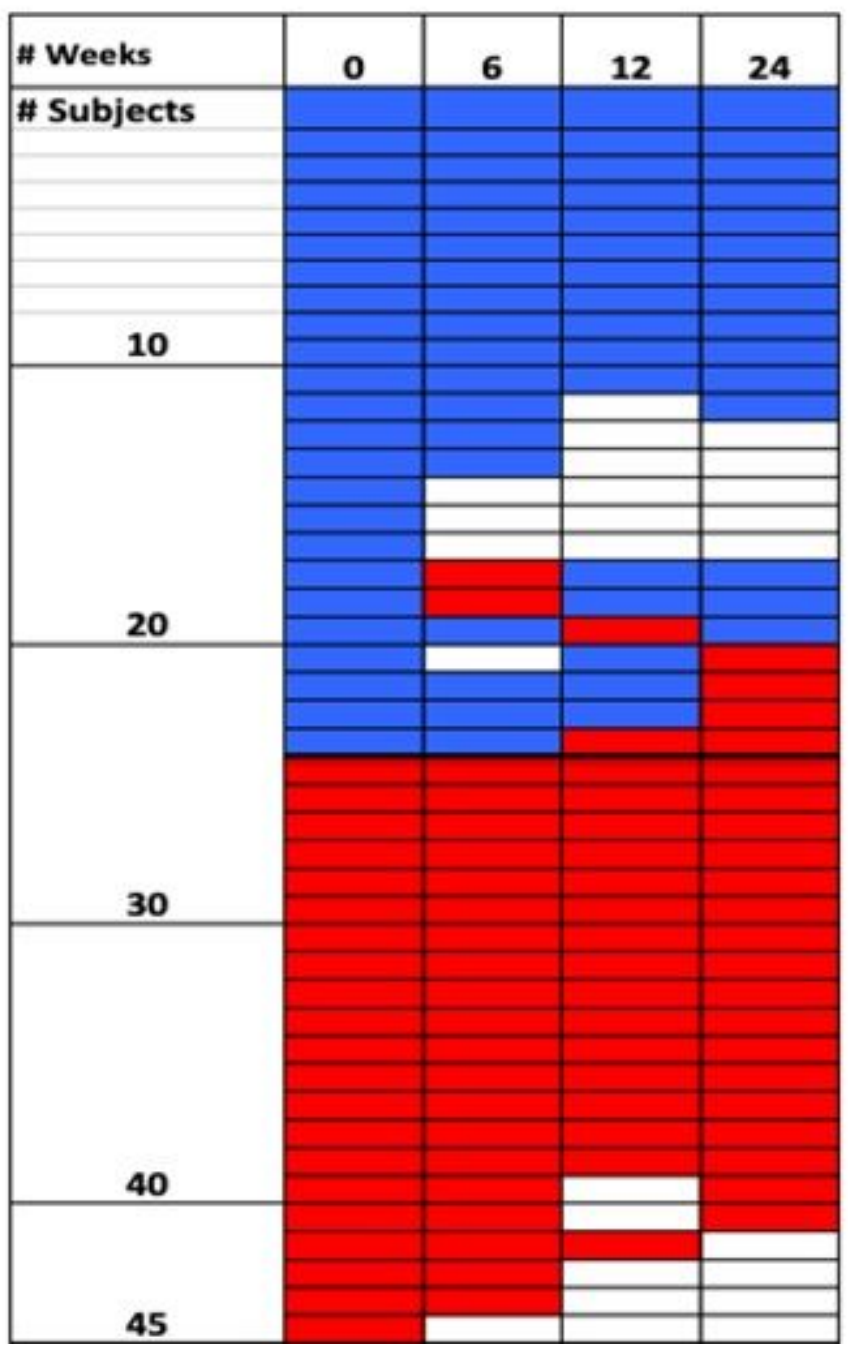

Antibiotic

treatment

B: Treatment Group $\downarrow$

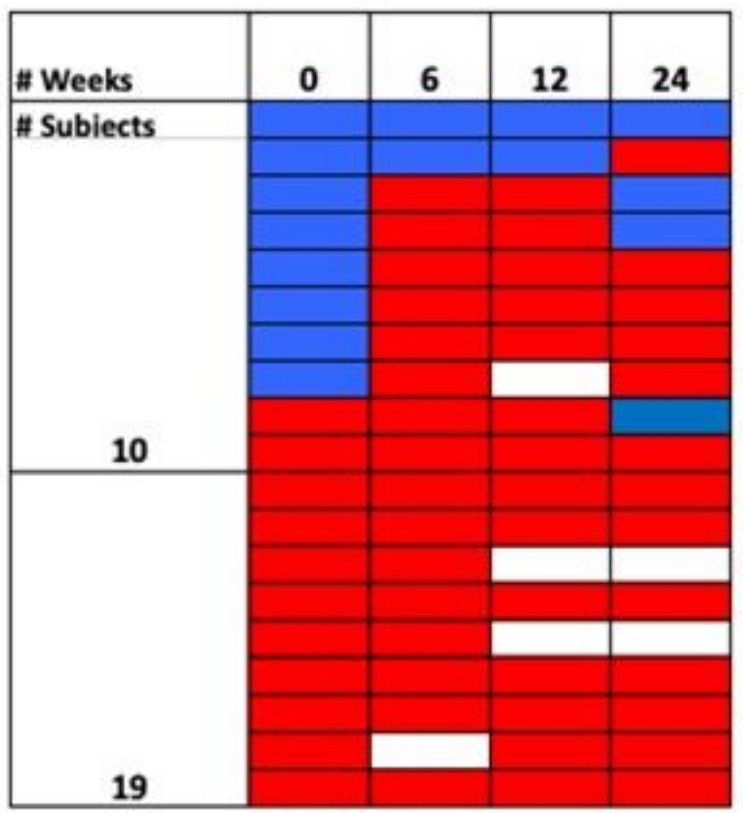

O. formigenes-positive

O. formigenes-negative NA

Figure 2

O. formigenes colonization status in 45 control subjects and 19 antibiotic-treated subjects over the study period. O. formigenes-positive samples are shown in blue, negative in red, and missing in white. Panel A: Untreated control subjects. Panel B: Antibiotic-treated subjects. 

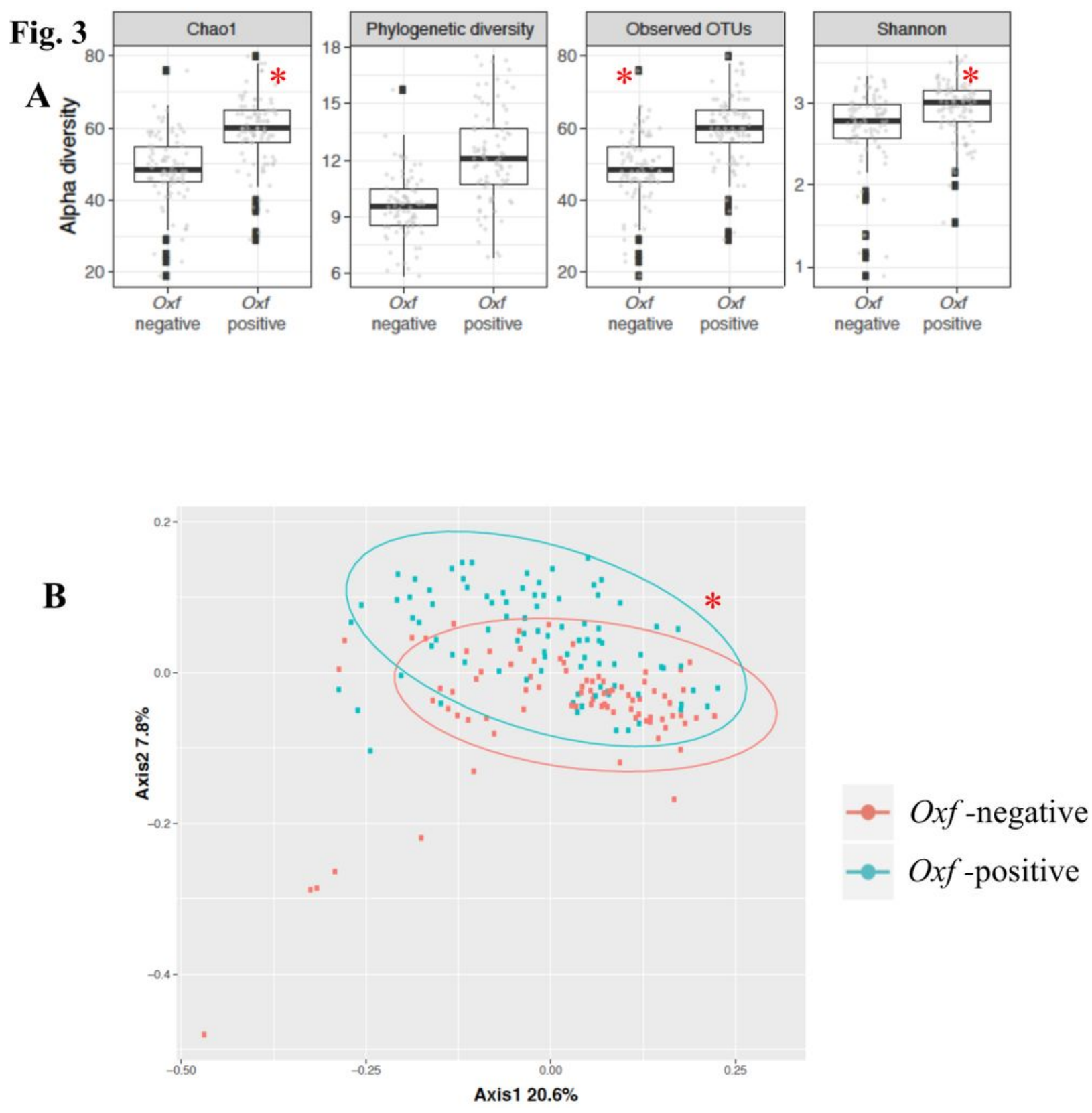

Figure 3

Alpha- and beta-diversity of microbiota in 173 fecal samples from 64 study subjects, according to baseline 0 . formigenes status. Panel A: Alpha-diversity measurements. Chao1, phylogenetic diversity, observed OTUs, and Shannon diversity according to Oxf status at baseline. Panel B: Analysis of Betadiversity, Unweighted UniFrac distances, according to 0 . formigenes status. * $p<0.05$ 
Fig. 4

A

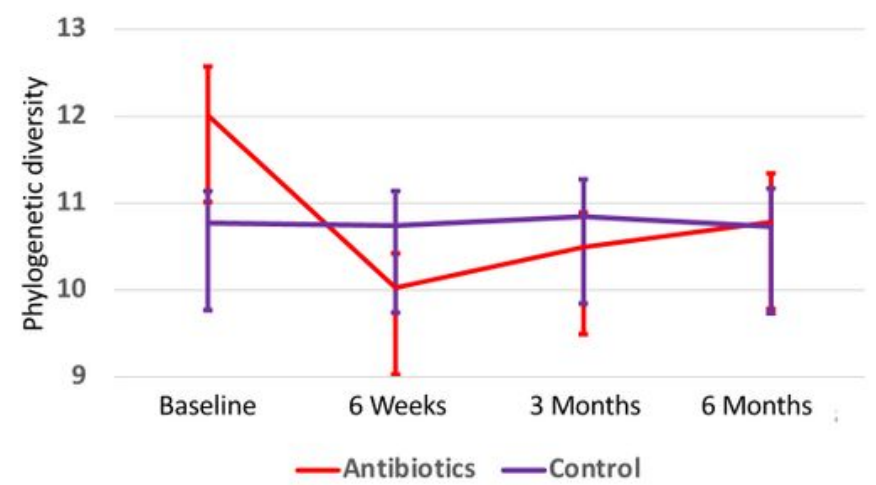

\begin{tabular}{|c|c|c|c|}
\hline Group 1 & Group 2 & p-value & $q$-value \\
\hline$A 0(n=19)$ & A6W $(n=17)$ & 0.008 & 0.024 \\
\hline$A 0(n=19)$ & A3M $(n=16)$ & 0.091 & 0.14 \\
\hline$A 0(n=19)$ & $\operatorname{A6M}(n=16)$ & 0.128 & 0.13 \\
\hline $\mathrm{CO}(n=44)$ & C6W (n=39) & 0.985 & 1.0 \\
\hline $\operatorname{CO}(n=44)$ & C3M $(n=35)$ & 0.875 & 1.0 \\
\hline $\mathrm{CO}(\mathrm{n}=44)$ & $\operatorname{c6} M(n=36)$ & 0.946 & 1.0 \\
\hline & \multicolumn{2}{|c|}{$\begin{array}{l}\text { A: Antibiotic-treatec } \\
\text { C: Controls } \\
\text { W: Weeks } \\
\text { M: Months }\end{array}$} & \\
\hline
\end{tabular}

Fig. 4

B
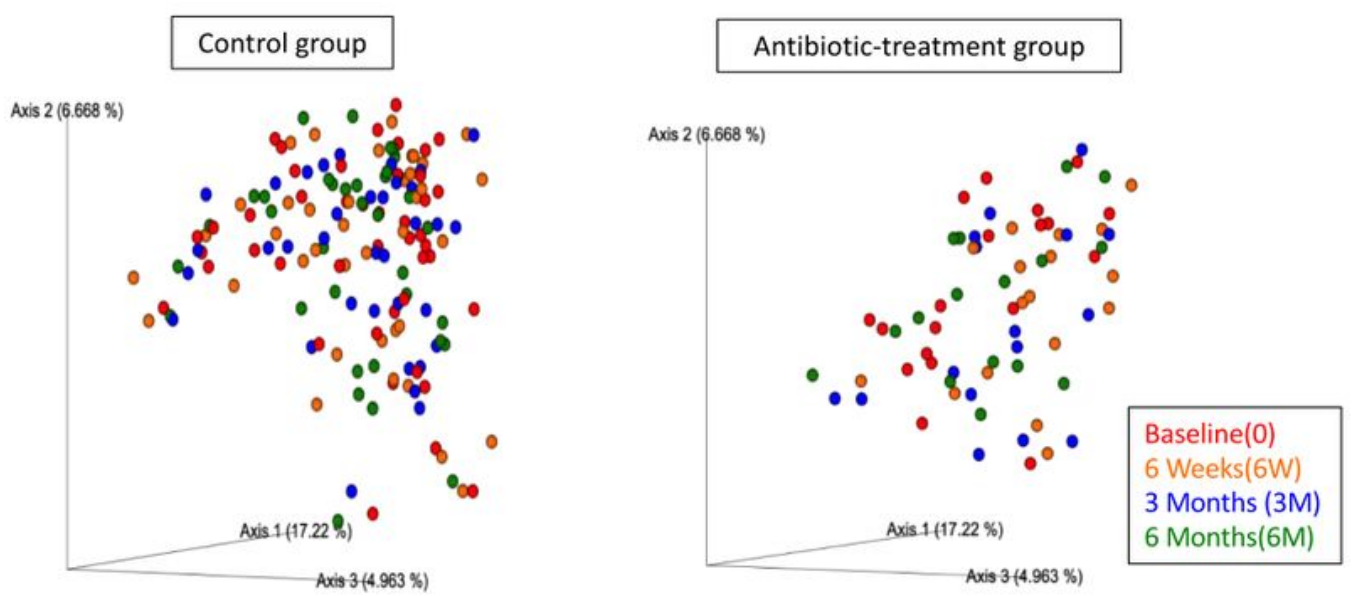

\begin{tabular}{|c|c|c|c|c|}
\hline Group 1 & Group 2 & Sample size & \# Perm & p-value \\
\hline C0 & C6W & 83 & 999 & 1 \\
\hline C0 & C3M & 79 & 999 & 1 \\
\hline C0 & C6M & 80 & 999 & 0.999 \\
\hline
\end{tabular}

\begin{tabular}{|c|c|c|c|c|}
\hline Group 1 & Group 2 & Sample size & \# Perm & p-value \\
\hline A0 & A6W & 36 & 999 & 0.06 \\
\hline A0 & A3M & 35 & 999 & 0.12 \\
\hline A0 & A6M & 35 & 999 & 0.54 \\
\hline
\end{tabular}

\section{Figure 4}

Alpha- and beta-diversity of microbiota in controls and antibiotic-treated subjects over time. Panel A: Phylogenetic diversity over time in the two groups of subjects; Kruskal Wallace analysis. Panel B: Unweighted UniFrac distances over time.

\section{Supplementary Files}


This is a list of supplementary files associated with this preprint. Click to download.

- Supplmaterial123020.docx 